\title{
Fatores modificáveis da degeneração macular relacionada à idade
}

\author{
Modifiable risk factors for age-related macular degeneration
}

\author{
Rogiil José de Almeida Torres ${ }^{1}$ \\ Maurício Maia² \\ Cristina Muccioli ${ }^{3}$ \\ Guilherme Winter ${ }^{4}$ \\ Greyce Kelly de Souza ${ }^{5}$ \\ Luca Rodrigo Pasqualotto ${ }^{6}$ \\ Andréa Luchini ${ }^{7}$ \\ Dalton Bertolim Précoma ${ }^{8}$
}

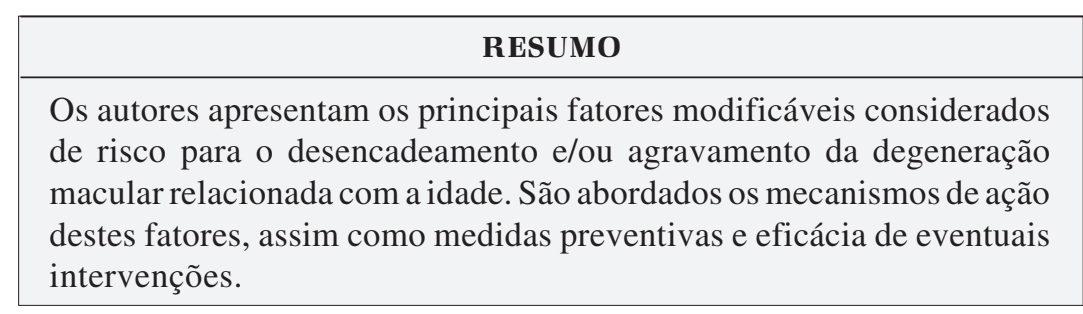

Descritores: Tabagismo; Hipertensão; Hipercolesterolemia; Aterosclerose; Índice de massa corporal; Raios ultravioleta; Luz; Antioxidantes; Carotenóides; Luteína; Ácidos graxos ômega-3; Dieta; Degeneração macular; Suplementação alimentar; Radicais livres: Ensaios clínicos controlados aleatórios como assunto
Trabalho realizado no Departamento de Oftalmologia da Universidade Federal de São Paulo - UNIFESP, no Hospital Angelina Caron - Campina Grande do Sul (PR) e na Pontifícia Universidade Católica do Paraná - PUCPR Curitiba (PR) - Brasil.

${ }^{1}$ Preceptor da Residência Médica do Hospital Angelina Caron, Campina Grande do Sul - (PR) - Brasil. Pósgraduando nível Doutorado do Departamento de Oftalmologia da Universidade Federal de São Paulo UNIFESP - São Paulo (SP) - Brasil.

${ }^{2}$ Orientador da pós-graduação do Departamento de Oftalmologia da UNIFESP - São Paulo (SP) - Brasil. Médico Oftalmologista Diretor do Serviço de Cirurgia Vitreorretiniana do Hospital de Olhos Oeste Paulista - Assis (SP) - Brasil.

${ }^{3}$ Professora livre docente Adjunta do Departamento de Oftalmologia da UNIFESP - São Paulo (SP) - Brasil.

${ }^{4}$ Acadêmico da Pontifícia Universidade Católica do

Paraná - PUCPR - Curitiba (PR) - Brasil.

${ }^{5}$ Acadêmica da PUCPR - Curitiba (PR) - Brasil.

${ }^{6}$ Acadêmico da PUCPR - Curitiba (PR) - Brasil.

${ }^{7}$ Médica do Centro Oftalmológico de Curitiba - Curitiba (PR) - Brasil.

${ }^{8}$ Professor Adjunto do Departamento de Cardiologia da PUCPR - Curitiba (PR) - Brasil.

Endereço para correspondência: Rogil José de Almeida Torres. Praça Rui Barbosa, 827 - Conj. 305 Curitiba (PR) CEP 80010-030

E-mail: rjat@terra.com.br

Recebido para publicação em 15.06.2008

Última versão recebida em 08.12 .2008

Aprovação em 17.12.2008

\section{INTRODUÇÃO}

A degeneração macular relacionada à idade (DMRI) é uma das principais causas de perda visual na terceira idade. Vários tratamentos propostos, que anunciavam grande expectativa de cura, caíram em desuso devido à ineficiência e complicações. Grandes somas financeiras têm sido investidas em pesquisas e ensaios clínicos para tentar atenuar os efeitos visuais devastadores da DMRI, entretanto o máximo que se consegue, na maioria dos casos, é protelar a perda da visão central. Sendo assim, este artigo visa chamar a atenção para a importância do reconhecimento dos fatores modificáveis da DMRI, ou seja, aqueles que têm o potencial de induzir, agravar ou prevenir tal patologia e que são passíveis de intervenção. Entre eles destacam-se: tabagismo, hipertensão arterial sistêmica, hipercolesterolemia, aterosclerose, circunferência abdominal, índice cintura/quadril, índice de massa corporal, raios ultravioleta, luz visível, dieta, antioxidantes sintéticos e atividade física. São fatores de grande relevância, pois dependem exclusivamente da mudança de hábito de vida. Sendo assim podem ser facilmente difundidos pelos médicos e adotados pelos pacientes.

\section{TABAGISMO}

Entre os fatores considerados de risco para a DMRI, tabagismo é considerado o mais importante ${ }^{(1)}$. A fisiopatologia no desenvolvimento desta doença não é totalmente conhecida, porém sugere-se que o cigarro diminua os níveis plasmáticos de antioxidantes, afetando de forma indireta o metabolismo coriorretiniano ${ }^{(2)}$. Além disso, o tabagismo promove a aterosclerose dos vasos da coróide podendo ocasionar atrofia geográfica (AG) e neovascularização sub-retiniana (NVSR) ${ }^{(1)}$.

Experimentalmente foi demonstrado que a nicotina, isolada ou em associação a fatores de crescimento, pode provocar neovascularização da co- 
róide ${ }^{(3)}$. Outro estudo demonstrou que o cigarro causa danos no epitélio pigmentário da retina (EPR), levando à formação de drusas, lesão da membrana de Bruch e consequente crescimento de neovasos sub-retinianos ${ }^{(4)}$. Estas alterações são provocadas pelo efeito direto da nicotina, que ativa os mediadores pró-inflamatórios e potencializa a proliferação das células musculares endoteliais ${ }^{(5)}$.

Outro mecanismo fisiopatológico sugerido é a presença diminuída do gene complementar fator $\mathrm{H}$ (CFH) nos tabagistas, o qual está associado a um alto risco de degeneração macular ${ }^{(6)}$.

Os estudos populacionais realizados até o momento são passíveis de erros metodológicos e estatísticos. Muitos correlacionam o tempo de exposição ao tabaco com o desenvolvimento da doença, enquanto outros defendem a carga tabágica como a mais importante na gênese da DMRI, criando um conflito entre os diversos autores. No entanto, há um consenso quanto à ligação entre o tabagismo e a DMRI avançada, ou seja, atrofia geográfia e neovascularização. Também se pode inferir que o tabagismo está pouco ou nada relacionado às formas iniciais de $\mathrm{DMRI}^{(7)}$.

Há quase um consenso em que o tempo de exposição ao tabaco, seja ele de forma ativa ou passiva, bem como a carga tabágica, são de grande importância no aumento do risco de desenvolvimento e incidência da doença. Fumantes ativos ou ex-fumantes, há menos de 20 anos, apresentam um risco muito maior de desenvolver a doença do que aqueles que cessaram o tabagismo há mais de 20 anos ou que nunca fumaram ${ }^{(8)}$.

\section{HIPERTENSÃO ARTERIAL SISTÊMICA}

A hipertensão arterial sistêmica (HAS), fator envolvido na doença cardiovascular, influencia na circulação coroidal ${ }^{(9)}$. Há estudos demonstrando que o controle da HAS pode diminuir o risco de DMRI ${ }^{(10)}$. A pressão de pulso, diferença entre pressão arterial sistólica (PAS) e pressão arterial diastólica (PAD), pode predispor a alterações do colágeno e elastina ocorridas na membrana de Bruch $^{(10)}$. Associações entre DMRI neovascular e níveis pressóricos elevados, $\mathrm{PAD}$ acima de $95 \mathrm{mmHg}$, já foram detectadas em estudos populacionais de caso-controle ${ }^{(10-11)}$, assim como a diminuição esteve associada à redução da DMRI inicial $^{(10)}$. Estudos demonstraram que o aumento da PAS e pressão de pulso aumentam o risco de desenvolver drusas e outros sinais da DMRI inicial e exsudativa ${ }^{(10,12)}$. Pacientes hipertensos tratados e que mantiveram um bom controle da PA tiveram duas vezes mais DMRI que as pessoas normotensas, enquanto que os hipertensos mal controlados tiveram incidência três vezes maior. Em contrapartida, um estudo envolvendo três grandes ensaios clínicos não correlacionou a HAS com a DMRI ${ }^{(13)}$.

\section{HIPERCOLESTEROLEMIA}

A elevação do colesterol sérico provoca acúmulo de lipídios na esclera aumentando a resistência pós-capilar da rede vascular da coróide, situada entre a parede escleral progressi- vamente enrijecida e o conteúdo não compressível do globo. A diminuição do fluxo sanguíneo da coróide e o aumento da resistência tendem a elevar a pressão hidrostática da coriocapilar aumentando o vazamento e deposição de proteínas extracelulares e lipídios, particularmente no pólo posterior, formando depósitos basais na membrana de Bruch e drusas, primeira manifestação clínica da DMRI ${ }^{(14)}$. Na década de 80 , após estudar o coeficiente de rigidez escleral de 29 pacientes com DMRI, esta teoria foi solidificada ${ }^{(14)}$.

Porém o aumento do colesterol sérico não pode ser considerado um fator de risco independente para degeneração macular. Geralmente, quando o nível de colesterol encontra-se elevado, há uma dieta rica em gorduras associada e, como consequência, a aterosclerose também aparece para contribuir com a gênese desta doença.

Um estudo populacional ${ }^{(15)}$ tentou comparar as lipoproteínas séricas aterogênicas e não-aterogênicas com o objetivo de responder à pergunta: o aumento do colesterol sérico é fator de risco para DMRI? Os autores recrutaram pacientes do serviço de oftalmologia da Universidade do Alabama, Estados Unidos, com mais de 50 anos e que possuíam acuidade visual de 20/80, ou melhor, em pelo menos um olho. Assim, 129 pacientes foram selecionados, sendo 72 mulheres e 57 homens. Além disso, foram mensuradas as apoliproteínas sanguíneas: apoB, a principal representante das lipoproteínas de baixa densidade (LDL), e apoA-I, a principal representante de alta densidade (HDL). Os autores não encontraram significância estatística nas relações entre as apolipoproteínas e o estágio da maculopatia. Assim, os níveis de apoB, a principal representante das lipoproteínas de baixa densidade (LDL), em pacientes sem maculopatia, sinais leves, intermediários e avançados de DMRI foram, respectivamente, 93,3, 91,8, 95,2 e 98,2 mg/dL. Mesmo não apresentando significância, os valores encontrados mostraram que colesterol sérico elevado está associado à doença avançada. Já com relação às lipoproteínas de alta densidade, os valores encontrados, para os mesmos grupos supracitados, foram 147,4, 148,6, 141,0 e 144,9. Estes dados mostraram que níveis mais altos de apoA-I, a principal representante da lipoproteína de alta densidade (HDL), estão relacionados aos sinais iniciais da doença ou à ausência da doença degenerativa macular, sugerindo efeito protetor das lipoproteínas pertencentes ao grupo do HDL. O colesterol total, mensurado nos mesmos grupos, foi de 201, 198, 194 e 203. Concluiu-se que altos níveis lipídicos no sangue podem desempenhar papel no desenvolvimento inicial da DMRI, ou seja, as drusas.

Um estudo caso-controle que avaliou 18.007 pacientes com diagnóstico de DMRI e 86.169 controles $^{(16)}$, concluiu não haver diferença estatística entre o uso de drogas redutoras do colesterol e DMRI, quando administrada por pouco tempo ( $<5$ anos). No entanto, a longo prazo ( $>5$ anos), o uso de estatinas diminuiu o risco de DMRI, principalmente a forma neovascular (risco relativo 0,51 quando comparada à forma seca ou atrófica). Desta forma sugere-se que as estatinas exercem fator protetor contra o desenvolvimento da doença macular degenerativa. 


\section{ATEROSCLEROSE}

Não foi demonstrada a associação entre aterosclerose periférica (achado pelo índice tornozelo-braço) e risco de maculopatia relacionada à idade ${ }^{(17)}$. Entretanto, ao aferir as placas de gordura centrais observou-se que o espessamento da parede da artéria carótida comum estava relacionada a significativo aumento do risco de DMRI. Sendo assim, foi formulada a hipótese que a estenose de vasos centrais ocasionaria diminuição da oferta de sangue para a artéria oftálmica e, consequentemente redução da circulação coroidal e retiniana. Os autores concluíram que a presença de aterosclerose subclínica pode promover desenvolvimento de drusas e outros sinais de doença degenerativa macular inicial ${ }^{(17)}$.

Outro estudo ${ }^{(18)}$ observou que placas ateroscleróticas na bifurcação da carótida estavam associadas a aumento da prevalência de DMRI em 4,5 vezes. As placas presentes na artéria carótida comum e nas artérias periféricas tiveram uma prevalência aumentada em 2 vezes. Sendo assim, ficou demonstrado forte associação entre a aterosclerose e DMRI na população mais idosa. Os autores sugeriram a realização de estudos complementares com o intuito de responder a seguinte questão: a aterosclerose é uma causa direta da DMRI ou ocorre concomitantemente a outros fatores de risco?

Outro estudo de cohorte multiracial ${ }^{(19)}$, avaliou a relação entre sinais precoces de degeneração macular relacionada à idade e aterosclerose subclínica. Observou-se que os achados clínicos de aterosclerose e DMRI foram diferentes entre os quatro grupos étnicos, o que sugere que a prevenção deve ser feita de forma individualizada em cada grupo.

\section{CIRCUNFERÊNCIA ABDOMINAL}

O padrão de distribuição de gordura corporal estabelece diferenças prognósticas mais importantes que o índice de massa corpórea (IMC) para doenças cardiovasculares e síndrome metabólica. Há dois tipos essenciais de distribuição da gordura corporal: 1 - padrão central, mais comum em homens, cuja adiposidade está distribuída preferencialmente no tronco e ocorre aumento da deposição intra-abdominal visceral; 2 tipo periférico, mais comum em mulheres, cuja adiposidade está preferencialmente em quadril, nádegas e coxas. Sabe-se que indivíduos que apresentam circunferência abdominal aumentada ( $>102 \mathrm{~cm}$ para homens e $>80 \mathrm{~cm}$ para mulheres), têm distribuição adiposa mais visceral, com risco para doenças cardiovasculares e síndrome metabólica. A confluência entre o aumento da circunferência abdominal e a DMRI pode apontar para a inflamação, fenômeno comum a ambas.

Um estudo prospectivo visou demonstrar a progressão da DMRI precoce e intermediária para a forma tardia associando a fatores de risco e estilo de vida, como IMC, circunferência abdominal, índice cintura/quadril, atividade física ${ }^{(20)}$. A medida da circunferência abdominal foi realizada por fita métrica e protocolos padronizados. Os altos valores para circunferência abdominal, ou seja, a obesidade visceral dobrou o risco de progressão da DMRI em comparação com o controle. Portanto, indivíduos com DMRI precoce ou intermediária, com distribuição de gordura visceral aumentada, têm risco aumentado de evoluir para DMRI avançada.

\section{ÍNDICE CINTURA/QUADRIL (ICQ)}

A relação cintura/quadril ICQ é definida pela divisão do perímetro abdominal (entre a última costela e a crista ilíaca) pelo perímetro do quadril (nível dos trocânteres femorais). Índice maior que 0,8 em mulheres e 0,9 em homens estão correlacionados com maior distribuição de gordura visceral, em detrimento da periférica, ou seja, maior risco de doença cardiovascular e síndrome metabólica. A associação entre este fator e a DMRI também está vinculada ao componente inflamatório.

Um estudo demonstrou que o aumento da gordura visceral aumenta o risco de progressão da DMRI precoce e evolução da DMRI intermediaria para a avançada ${ }^{(20)}$.

\section{ÍNDICE DE MASSA CORPORAL}

O índice de massa corporal (IMC) é utilizado para avaliar o grau de obesidade. O IMC é calculado dividindo o peso, em quilogramas, pela altura, em metros ao quadrado. O componente inflamatório, muitas vezes associado à obesidade e às doenças cardiovasculares, pode ser o elemento comum entre IMC e DMRI.

Um estudo prospectivo que dividiu o IMC em três categorias, $<25,25-29$ e $>30 \mathrm{~kg} / \mathrm{m}^{2}$ demonstrou que elevado IMC aumenta o risco de progressão da DMRI. Risco relativo de 2,32 para IMC entre $25-29 \mathrm{~kg} / \mathrm{m}^{2}$; de 2,35 para IMC maior que $30 \mathrm{~kg} / \mathrm{m}^{2}$, enquanto que IMC menor que $25 \mathrm{~kg} / \mathrm{m}^{2}$ não apresentou associação com DMRI ${ }^{(20)}$.

Outro estudo, prospectivo de caso-controle, demonstrou que o aumento do IMC estava associado com a presença de DMRI neovascular ${ }^{(12)}$.

Entretanto, em outro relato que combinou três estudos de populações diferentes (The Beaver Dam Eye Study [BDES], the Blue Mountains Eye Study [BMES] e the Rotterdam Study [RS]) para examinar os fatores de risco que interferem com a incidência de DMRI, não foi observada associação do IMC com a incidência de DMRI ${ }^{(13)}$.

\section{RAIOS ULTRAVIOLETA E LUZ VISÍVEL}

Com a idade o número de células do EPR diminui, particularmente no centro da mácula, assim como a densidade de melanossomos contidos no seu interior. Sabe-se que a melanina desempenha importante papel na remoção de radicais livres e é responsável pela absorção da radiação luminosa ${ }^{(21)}$. Observa-se também contínuo aumento da lipofuscina intra- 
celular no EPR que leva à deterioração da função celular, tornando a retina mais sensível aos raios luminosos. Os grânulos de lipofuscina apresentam-se em maior número que os grânulos de melanina e comumente ocorre fusão destes, originando a melanofuscina. Estes grânulos ocupam substancial espaço citoplasmático que induzem gradativamente a morte celular. Em áreas com elevada concentração de lipofuscina pode-se observar atrofia do EPR e consequente desenvolvimento da DMRI.

Há estudos que demonstram não haver associação entre DMRI e exposição crônica aos raios ultravioletas A ou B. Sabe-se que a córnea protege a retina dos raios ultravioletas menores que $300 \mathrm{~nm}$, enquanto que o cristalino bloqueia raios UV entre 300 e $400 \mathrm{~nm}^{(22)}$. Por outro lado, há evidências experimentais demonstrando que a exposição à luz azul pode contribuir para o desenvolvimento da DMRI ${ }^{(23-24)}$. Tanto a luz violeta quanto a luz azul apresentam importante toxicidade retiniana, porém a luz violeta apresenta pouco efeito na visão escotópica enquanto que a luz azul, além de desempenhar importantes funções biológicas retinianas, é essencial para este tipo de visão. É importante lembrar que a luz violeta apresenta comprimentos de ondas entre $400-440 \mathrm{~nm}$ e a luz azul entre $440-500 \mathrm{~nm}$.

Os fótons alto-energéticos, no espectro da luz violeta, azul e radiação UVA, têm poder para danificar a estrutura e função celular dos fotorreceptores e EPR. Estes fótons criam espécies reativas de oxigênio, que são nocivas a várias organelas celulares, particularmente o DNA mitocondrial, resultando na morte celular apoptótica ${ }^{(23-24)}$.

Quanto menor o comprimento de onda da radiação, maior será a formação de espécies reativas de oxigênio. Em nível celular, o comprimento de onda entre 470-490 induz injúria oxidante em ambos os segmentos externos de cones e bastonetes, num evento que exige a ativação da rodopsina. Além do que a luz azul tem o potencial de aumentar o número de moléculas rodopsina ${ }^{(25)}$, agravando o quadro.

Enquanto que, experimentalmente, consegue-se com relativa facilidade demonstrar os efeitos nocivos causados pela radiação luminosa de ondas curtas, o mesmo não ocorre para comprová-los em estudos epidemiológicos. É difícil determinar a exposição luminosa cumulativa em cada indivíduo retrospectivamente, além do que a DMRI é uma doença multifatorial. Dois grandes estudos populacionais acharam correlação entre exposição cumulativa à luz e DMRI ${ }^{(26)}$, porém outros não encontraram tal correlação $0^{(27-28)}$.

Preventivamente, há estudos demonstrando que o uso de chapéu e óculos de sol entre a $2^{\underline{a}}$ e $3^{\underline{a}}$ década de vida, em pessoas com alta exposição luminosa, poderia reduzir a incidência de drusas moles e despigmentação do $\mathrm{EPR}^{(28)}$. Outro efeito protetor seria a utilização de lentes intraoculares, nas cirurgias de catarata, com filtro contra a luz azul, porém é importante frisar que este tipo de filtro limita significativamente a visão escotópica e que novo modelo de lente, filtrando somente a luz violeta, seria mais conveniente ${ }^{(29)}$.

\section{DIETA}

Sabe-se que a dieta é um dos meios mais eficientes para a atuação preventiva no desenvolvimento da DMRI. Há vários estudos relacionando os aspectos dietéticos da população com os tipos e fases da degeneração macular. Os resultados sugerem que dietas ricas em nutrientes antioxidantes, zinco e peixe ou ácidos graxos ômega-3 estão associados a taxas mais baixas da DMRI. Foi recomendado o uso de vitamina E, através do consumo de grãos (cereais, trigo, cevada), óleo vegetal, ovos e nozes, enquanto que o zinco está presente nos grãos, laticínios, carne, aves e peixes. Este autor lembra que a cenoura, couve, repolho e espinafre são os principais suplementos do beta-caroteno, enquanto a vitamina $\mathrm{C}$ é encontrada em frutas cítricas, pimenta verde, brócolis e batatas. A luteína e zeaxantina estão presentes em frutas e legumes. Este artigo afirma que alimentos contendo tais nutrientes são mais importantes que suplementos nutricionais ${ }^{(30)}$.

Por outro lado, elevado consumo de gorduras e de alimentos com alto índice glicêmico estiveram associados à maior prevalência e incidência da doença avançada (tardia). Um estudo envolvendo 3.654 pessoas acima de 49 anos demonstrou que a ingestão de gorduras, principalmente saturadas, e de colesterol, aumentam em $80 \%$ e $60 \%$, respectivamente, o risco de desenvolvimento de degeneração macular relacionada à idade ${ }^{(31)}$.

Foi observado que a ingestão de alimentos com alto índice glicêmico (IG) possibilita o desenvolvimento de DMRI 2,71 vezes mais que o consumo de dieta com baixo índice glicêmico (frutas, cereais, verduras, pães integrais) ${ }^{(32)}$. Da mesma forma, a ingestão total de gordura está associada a maior risco de desenvolvimento da doença, enquanto que o consumo de peixe 4 vezes por semana ou mais apresentou menor risco de DMRI. A relação inversa não aconteceu com a ingestão aumentada de ácido linoléico. $\mathrm{O}$ estudo chegou à conclusão que a ingestão total de gorduras tem alta influência na gênese da DMRI e que o ácido linoléico, gordura poli-insaturada, promove maior dano oxidativo macular ${ }^{(33)}$.

Eye Disease Case-Control Study ${ }^{(34)}$ avaliou a relação entre a ingestão total de gordura e degeneração macular relacionada à idade. Os resultados revelaram que a alta ingestão de gordura vegetal aumenta em 2,2 vezes a possibilidade de desenvolvimento de degeneração macular. Houve significativa associação entre DMRI e gorduras saturadas $(\mathrm{p}=0,04)$, monoinsaturadas $(\mathrm{p}<0,001)$ e gorduras poli-insaturadas $(\mathrm{p}=0,009)$. Dado interessante revelou não haver significância estatística entre ingestão de gordura animal e DMRI. No entanto, a ingestão de gordura vegetal foi estatisticamente significativa $(\mathrm{p}=0,006)$. Assim, uma dieta que substitua carboidratos por gordura vegetal está positivamente associada ao risco de degeneração macular. Observou-se também que o ácido linoléico aumenta significativamente o risco da doença $(\mathrm{p}=0,004)$. Por outro lado, a ingestão de ácidos graxos do tipo ômega-3 teve ação contrária ao ácido linoléico, ou seja, diminuiu o risco de DMRI. A ingestão regular de peixes mostrou proteção contra a DMRI, mas apenas nos indivíduos com baixo consumo de ácido lino- 
léico. Desta forma, o estudo concluiu que não apenas a ingestão total de gorduras, mas também dietas baseadas em certos tipos de gorduras podem aumentar o risco de DMRI, enquanto outras podem diminuir o risco da doença.

Outro estudo demonstrou que o consumo de ômega-3 diminui o risco de DMRI e que a ingestão de peixes, pelo menos uma vez por semana, pode reduzir em $40 \%$ o risco da doença precoce ${ }^{(32)}$. Observou-se também que os participantes que consumiram peixe mais de 3 vezes na semana tiveram redução do risco da doença tardia. Quanto ao ácido linoléico, a ingestão de altas doses demonstrou aumentar o risco de DMRI. Participantes do grupo de menor consumo total de gorduras apresentaram risco $70 \%$ menor de doença precoce que aqueles que ingeriram alta quantidade. $\mathrm{O}$ estudo concluiu que dieta rica em gorduras poli-insaturadas, especialmente o ômega-3 proveniente dos peixes, reduziu o risco do desenvolvimento da DMRI, tanto precoce como tardia ${ }^{(31)}$.

O AREDS - Age-Related Eye Disease Study Research Group, relato $\mathrm{n}^{\circ} 20$, encontrou relação inversa entre alta ingestão de gorduras do tipo poli-insaturadas ômega-3 e DMRI do tipo neovascular $^{(35)}$. Resultado semelhante foi encontrado com o alto consumo de peixes, seja ele assado ou frito. Pelo contrário, dietas ricas em ácido araquidônico (ácido graxo poli-insaturado n-6) aumentaram significativamente o risco de DMRI neovascular, provavelmente por ser precursor de mediadores inflamatórios. Assim, como os outros artigos, este também concluiu que o consumo de peixes e alimentos ricos em ômega-3 diminui o risco de degeneração macular avançada, particularmente da forma neovascular.

\section{ANTIOXIDANTES SINTÉTICOS}

Antioxidantes são substâncias capazes de prevenir os efeitos deletérios da oxidação, inibindo o início da lipoperoxidação, sequestrando radicais livres e/ou quelando íons metálicos. Eles protegem organismos aeróbicos do estresse oxidativo.

Sabe-se que a retina pode gerar espécies reativas de oxigênio por diversas razões: alto consumo de oxigênio, possibilidade de altos níveis cumulativos de irradiação, sobretudo a luz azul e ultravioleta, presença de ácidos graxos (segmentos externos dos fotorreceptores), presença de cromóforos na retina neurossensorial e EPR, além do processo de fagocitose das extremidades de cones e bastonetes pelo EPR.

Pelo consenso que há a respeito do papel patogenético do dano oxidativo cumulativo na $\mathrm{DMRI}^{(36)}$, vários artigos têm sido publicados demonstrando eventuais efeitos benéficos do uso destas substâncias contra esta patologia ocular. Evidências clínicas sugerem que a inclusão de compostos antioxidantes como a vitamina $\mathrm{E}$, vitamina $\mathrm{A}$, vitamina $\mathrm{C}$ e o zinco, presentes em alguns alimentos, são capazes de prevenir e minimizar os efeitos da DMRI ${ }^{(37)}$. Entretanto, o relato $\mathrm{n}^{\circ} 22$ do AREDS - Age-Related Eye Disease Study Research Group ${ }^{(38)}$, que contou com 4.519 participantes, entre 60 e 80 anos de idade e avaliou a relação entre dieta com carotenóides, vitamina $\mathrm{A}$, alpha-tocoferol e vitamina $\mathrm{C}$ na DMRI demonstrou que a ingesta destes componentes isoladamente não têm correlação com esta doença.

Quanto a luteína e zeaxantina, há evidências demonstrando o potencial de aumentar a densidade de pigmento macu$\operatorname{lar}^{(39)}$. Sabe-se que os pigmentos são responsáveis pela filtragem e absorção da luz azul, atenuando o estresse oxidativo e protegendo consequentemente a retina. Entretanto, seus efeitos benéficos na degeneração macular permanecem controversos. O Food and Drug Administration (FDA) conduziu uma revisão de estudos para averiguar e ter a certeza sobre as evidências científicas do papel da luteína e zeaxantina na degeneração macular e cataratas ${ }^{(40)}$. Com base nas revisões, o FDA concluiu que não existem evidências fidedignas sobre a atuação da luteína e zeaxantina sobre o risco de degeneração macular e/ou catarata ${ }^{(41)}$. Finalmente o AREDS 2, estudo multicêntrico randomizado duplo-cego, que envolve 4.000 participantes entre 50 e 85 anos, tem por objetivo avaliar o efeito de suplementação oral com luteína/zeaxantina e/ou ácidos graxos poli-insaturados de cadeia longa ômega-3 (EPA e DHA) na progressão da DMRI avançada. Foi iniciado em setembro de 2006 e deverá esclarecer eventuais dúvidas destas substâncias na prevenção da DMRI (www.clinicaltrials.gov).

\section{ATIVIDADE FÍSICA}

Um estudo prospectivo objetivou mostrar a progressão da DMRI precoce e intermediária para a forma tardia associando-a a fatores de risco e estilo de vida, como IMC, circunferência abdominal, índice cintura/quadril, atividade física. Participaram do estudo, 261 pessoas acima de 60 anos, com acuidade visual de 20/200, ou melhor, em pelo menos um dos olhos, e que não tivessem DMRI exsudativa. O seguimento médio do estudo foi de 4,6 anos. Os participantes foram indagados através do questionário sobre o hábito de atividade física regular, e se sim, qual o número de vezes por semana isto acontecia. Os resultados foram compatíveis com uma redução de $25 \%$ no risco de progressão da DMRI precoce e intermediária para DMRI avançada entre aqueles que praticavam regularmente, 3 vezes por semana, atividade física em comparação àqueles que não praticavam ${ }^{(20)}$.

Outro estudo de cohort realizado com moradores de Beaver Dam objetivou determinar a associação entre doença cardiovascular e seus fatores de risco com a incidência de DMRI. A mostra foi de 3.684 pessoas que participaram em 5 anos e 2.764 que participaram os 10 anos. A faixa etária variou entre 43 a 86 anos. Foi observado que, independentemente da idade e outros fatores de risco, a atividade física está inversamente associada à progressão de AMD e a incidência de degeneração macular exsudativa ${ }^{(10)}$.

\section{CONCLUSÃO}

Como prevenção da degeneração macular relacionada à idade, além de cessar o hábito de fumar, convém aumentar o 
consumo de peixes e ingestão de ômega-3, bem como manter o HDL elevado e o LDL reduzido. Valores tensóricos normais e atividade física além de beneficiar o sistema cardiovascular podem exercer influência positiva na preservação da visão. $\mathrm{O}$ uso de antioxidantes sintéticos tem o seu valor quando a dieta alimentar composta por vitaminas, luteína e zeaxantina é deficiente. O ideal seria dosar laboratorialmente a quantidade de vitaminas presentes no organismo e repô-las se houver necessidade.

\section{ABSTRACT}

The authors present the main modifiable risk factors that may trigger and/or worsen age-related macular degeneration. Mechanisms of action related to these factors as well as preventive measures and intervention effectiveness are discussed.

Keywords: Smoking; Hypertension; Hypercholesterolemia; Atherosclerosis; Body mass index; Ultraviolet rays; Light; Antioxidants; Carotenoids; Lutein; Fatty acids, omega-3; Diet; Macular degeneration; Supplementary feeding; Free radicals; Randomized controlled trials as topic

\section{REFERÊNCIAS}

1. Vingerling JR, Hofman A, Grobbee DE, de Jong PT. Age-related macular degeneration and smoking. The Rotterdam Study. Arch Ophthalmol. 1996; 114(10):1193-6. Comment in: Arch Ophthalmol. 1997;115(7):945.

2. Stryker WS, Kaplan LA, Stein EA, Stampfer MJ, Sober A, Willett WC.The relation of diet, cigarette smoking, and alcohol consumption to plasma betacarotene and alpha-tocopherol levels. Am J Epidemiol. 1988;127(2):283-96.

3. Suñer IJ, Espinosa-Heidmann DG, Marin-Castano ME, Hernandez EP, PereiraSimon S, Cousins SW. Nicotine increases size and severity of experimental choroidal neovascularization. Invest Ophthalmol Vis Sci. 2004;45(1):311-7.

4. Bailey TA, Kanuga N, Romero IA, Greenwood J, Luthert PJ, Cheetham ME. Oxidative stress affects the junctional integrity of retinal pigment epithelial cells. Invest Ophthalmol Vis Sci. 2004;45(2):675-84.

5. Sastry BV, Hemontolor ME. Influence of nicotine and cotinine on retinal phospholipase A2 and its significance to macular function. J Ocul Pharmacol Ther. 1998;14(5):447-58.

6. Esparza-Gordillo J, Soria JM, Buil A, Almasy L, Blangero J, Fontcuberta J, et al. Genetic and environmental factors influencing the human factor $\mathrm{H}$ plasma levels. Immunogenetics. 2004;56(2):77-82.

7. Tan JS, Mitchell P, Kifley A, Flood V, Smith W, Wang JJ. Smoking and the long-term incidence of age-related macular degeneration: The Blue Mountains Eye Study. Arch Ophthalmol. 2007;125(8):1089-95.

8. Khan JC, Thurlby DA, Shahid H, Clayton DG, Yates JR, Bradley M, Moore AT, Bird AC; Genetic Factors in AMD Study. Smoking and age related macular degeneration: the number of pack years of cigarette smoking is a major determinant of risk for both geographic atrophy and choroidal neovascularisation. $\mathrm{Br}$ J Ophthalmol. 2006;90(1):75-80.

9. Metelitsina TI, Grunwald JE, DuPont JC, Ying GS. Effect of systemic hypertension on foveolar choroidal blood flow in age related macular degeneration. $\mathrm{Br}$ J Ophthalmol. 2006;90(3):342-6.

10. Klein R, Klein BE, Tomany SC, Cruickshanks KJ. The association of cardiovascular disease with the long-term incidence of age-related maculopathy: the Beaver Dam Eye Study. Ophthalmology. 2003;110(6):1273-80. Comment in: Am J Ophthalmol. 2007;144(3):482-3; author reply 483. Corrected and republished from: Ophthalmology. 2003;110(4):636-43.

11. van Leeuwen R, Ikram MK, Vingerling JR, Witteman JC, Hofman A, de Jong PT. Blood pressure, atherosclerosis, and the incidence of age-related maculopathy: The Rotterdam Study. Invest Ophthalmol Vis Sci. 2003;44(9):3771-7.

12. Age-Related Eye Disease Study Research Group. Risk factors associated with age-related macular degeneration. A case-control study: Age-Related Eye Disease Study Report Number 3. Ophthalmology. 2000;107(12):2224-32.

13. Smith W, Assink J, Klein R, Mitchell P, Klaver CC, Klein BE, et al. Risk factors for incident age-related macular degeneration: Pooled findings from three continents. Ophthalmology. 2001;108(4):697-704.

14. Friedman E, Ivry M, Ebert E, Glynn R, Gragoudas E, Seddon J. Increased scleral rigidity and age-related macular degeneration. Ophthalmology. 1989;96(1):104-8.

15. Dashti N, McGwin G, Owsley C, Curcio CA. Plasma apolipoproteins and risk for age related maculopathy. Br J Ophthalmol. 2006;90(8):1028-33. Erratum: Br J Ophthalmol. 2007;91(3):403.

16. Smeeth L, Cook C, Chakravarthy U, Hubbard R, Fletcher AE. A case control study of age related macular degeneration and use of statins. Br J Ophthalmol. 2005;89(9):1171-5

17. van Leeuwen R, Ikram MK, Vingerling JR, Witteman JC, Hofman A, de Jong PT. Blood pressure, atherosclerosis, and the incidence of age-related maculopathy: the Rotterdam Study. Invest Ophthalmol Vis Sci. 2003;44(9):3771-7.

18. Vingerling JR, Dielemans I, Bots ML, Hofman A, Grobbee DE, de Jong PT. Age-related macular degeneration is associated with atherosclerosis: the Rotterdam Study. Am J Epidemiol. 1995;142(4):404-9.

19. Klein R, Klein BE, Knudtson MD, Cotch MF, Wong TY, Liu K, et al. Subclinical atherosclerotic cardiovascular disease and early age-related macular degeneration in a multiracial cohort: the Multiethnic Study of Atherosclerosis. Arch Ophthalmol. 2007;125(4):534-43.

20. Seddon JM, Cote J, Davis N, Rosner B. Progression of age-related macular degeneration. association with body mass index, waist circumference, and waisthip ratio. Arch Ophthalmol. 2003;121(6):785-92.

21. Holz FG, Paulenikhoff D, Klein R, Bird AC. Pathogenesis of lesions in late age-related macular disease. Am J Ophthalmol. 2004;137(3):504-10.

22. Boettner EA, Wolter JR. Transmission of the ocular media. Invest Ophthalmol. 1962;1:776-83.

23. Grimm C, Wenzel A, Williams TP, Rol P, Hafezi F, Remé CE. Rhodopsinmediated blue light damage to the rat retina: effect of photoreversal bleaching. Invest Ophthalmol Vis Sci. 2001:42(2);497-505.

24. Wenzel A, Grimm C, Samardzija M, Remé CE. Molecular mechanisms of light-induced photoreceptor apoptosis and neuroprotection for retinal degeneration. Prog Retin Eye Res. 2005;24(2):257-306.

25. Van Gelder RN. Blue light and the circadian clock. Br J Ophthalmol. 2004; 88(10):1353; author reply 1353. Comment on: Br J Ophthalmol. 2003;87(12): 1523-9.

26. Tomany SC, Cruickshanks KJ, Klein R, Klein BE, Knudtson MD. Sunlight and the 10-year incidence of age-related maculopathy: the Beaver Dam Eye Study. Arch Ophthalmol. 2004;122(5):750-7. Erratum in: Arch Ophthalmol. 2005;123(3):362.

27. Delcourt C, Carriere I, Ponton-Sanchez A, Fourrey S, Lacroux A, Papoz L; POLA Study Group. Light exposure and the risk of age-related macular degeneration: the Pathologies Oculaires Liées a l'Age (POLA) study. Arch Ophthalmol. 2001;119(10):1463-8.

28. McCarty CA, Mukesh BN, Fu CL, Mitchell P, Wang JJ, Taylor HR. Risk factors for age-related maculopathy: the Visual Impairment Project. Arch Ophthalmol. 2001;119(10):1455-62.

29. Mainster MA. Intraocular lenses should block UV radiation and violet but not blue light. Arch Ophthalmol. 2005;123(4):550-5. Comment on: Arch Ophthalmol. 2005; 123(4):547-9.

30. van Leeuwen R, Boehhoorn S, Vingerling JR, Witteman JC, Klaver CC, Hofman A, De Jong PT. Dietary intake of antioxidants and risk of age-related macular degeneration. JAMA. 2005;294(24):3101-7.

31. Chua B, Flood V, Rochtchina E, Wang JJ, Smith W, Mitchell P. Dietary fatty acids and the 5-year incidence of age-related maculopathy. Arch Ophthalmol. 2006;124(7):981-6.

32. Chiu CJ, Hubbard LD, Armstrong J, Rogers G, Jacques PF, Chylack LT Jr, et al. Dietary glycemic index and carbohydrate in relation to early age-related macular degeneration. Am J Clin Nutr. 2006;83(4):880-6. Comment in: Am J Clin Nutr. 2006;83(4):733-4.

33. Cho E, Hung S, Willett WC, Spiegelman D, Rimm EB, Seddon JM, et al. Prospective study of dietary fat and the risk of age-related macular degeneration. Am J Clin Nutr. 2001;73(2):209-18.

34. Seddon JM, Rosner B, Sperduto RD, Yannuzzi L, Haller JA, Blair NP, et al. Dietary fat and risk for advanced age-related macular degeneration. Arch Ophthalmol. 2001;119(8):1191-9.

35. SanGiovanni JP, Chew EY, Clemons TE, Davis MD, Ferris FL $3^{\text {rd }}$, Gensler GR, Kurinij N, Lindblad AS, Milton RC, Seddon JM, Sperduto RD; AgeRelated Eye Disease Study Research Group. The relationship of dietary lipid intake and age-related macular degeneration in a case-control study: AREDS Report № 20. Arch Ophthalmol. 2007;125(5):671-9. 
36. Beatty S, Koh H, Phil M, Henson D, Boulton M. The role of oxidative stress in the pathogenesis of age-related macular degeneration. Surv Ophthalmol. 2000;45(2):115-34.

37. Seddon JM, Ajani UA, Sperduto RD, Hiller R, Blair N, Burton TC, et al. Dietary carotenoids, vitamins $\mathrm{A}, \mathrm{C}$, and $\mathrm{E}$, and advanced age related macular degeneration. Eye Disease Case-Control Study Group. JAMA. 1994;272(18):1413-20. Erratum in: JAMA. 1995;273(8):622. Comment in: JAMA. 1994;272(18):1455-6.

38. Age-Related Eye Disease Study Research Group, SanGiovanni JP, Chew EY, Clemons TE, Ferris FL $3^{\text {rd }}$, Gensler G, Lindblad AS, Milton RC, Seddon JM, Sperduto RD. The relationship of dietary carotenoid and vitamin A, E, and C intake with age-related macular degeneration in a case-control study. AREDS report $\mathrm{n}^{\circ}$ 22. Arch Ophthalmol. 2007;125(9):1225-32. Comment in: Arch Ophthalmol. 2008;125(9):1225-32.
39. Trieschmann M, Beatty S, Nolan JM, Hense HW, Heimes B, Austermann U, Fobker M, Pauleikhoff D. Changes in macular pigment optical density and serum concentrations of its constituent carotenoids following supplemental lutein and zeaxanthin: LUNA study. Exp Eye Res. 2007;84(4):718-28.

40. Trumbo PR, Ellwood KC. Lutein and zeaxanthin intakes and risk of age-related macular degeneration and cataracts: an evaluation using the Food and Drug Administration's evidence-based review system for health claims. Am J Clin Nutr. 2006;84(5):971-4.

41. U.S. Food and Drug Administration. Center for Food Safety and Applied Nutrition. Guidance for industry significant scientific agreement in the review of health claims for conventional foods and dietary supplements [Internet]. Rockville: Food and Drug Administration; 2000. [cited 2009 Fev 12]. Available from: http://www.cfsan.fda.gov/ dms/ssaguide.html

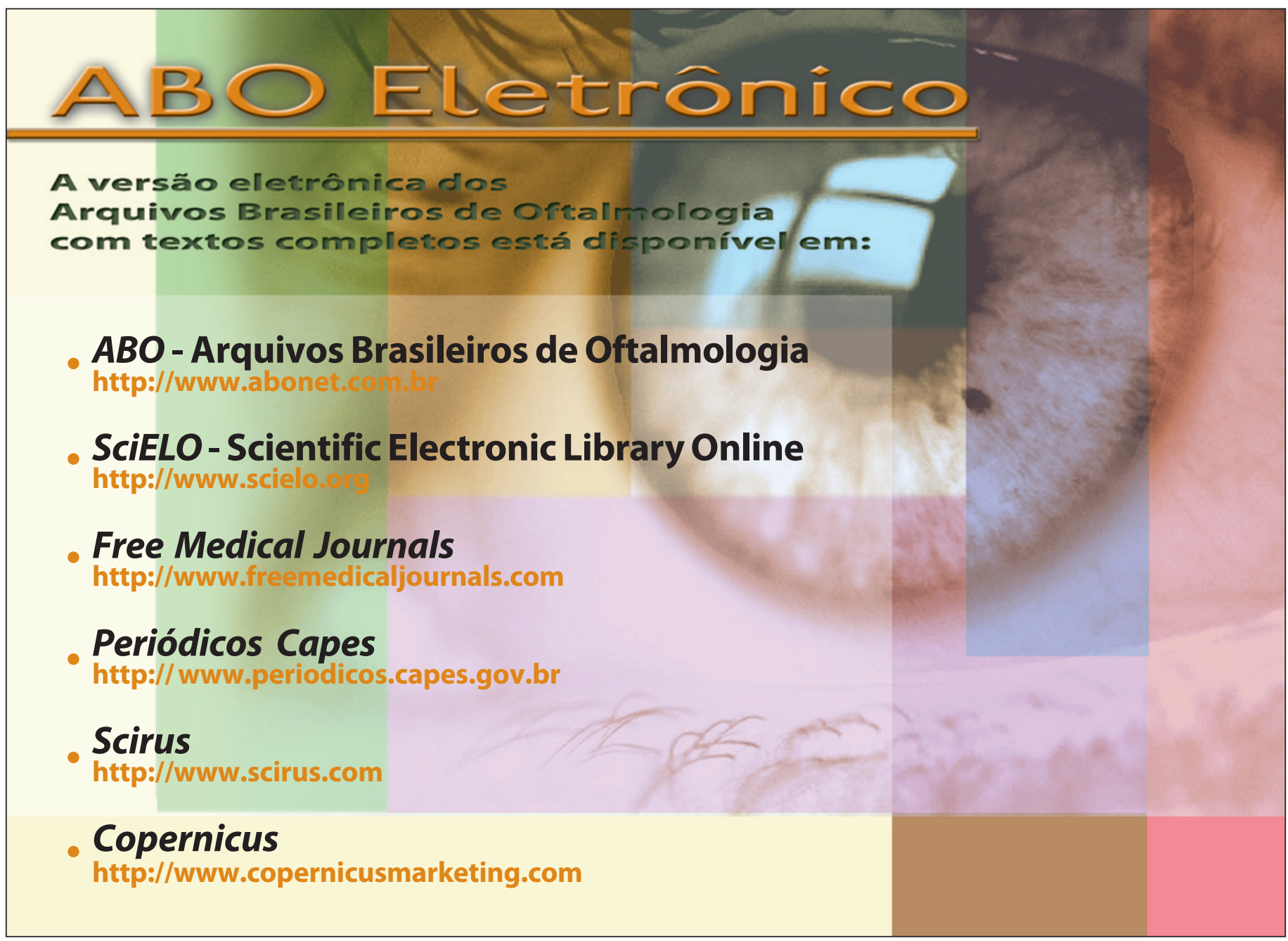

